\title{
La transformación de un programa de deporte escolar a través de lametodología comunicativa crítica: El consejo asesor \\ Transformation of an extracurricular sport programme through the critical communicative methodology: The advisory committee \\ Borja Jiménez Herranz, Víctor M. López Pastor y Juan Carlos Manrique Arribas \\ E.U. de Magisterio de Segovia (Universidad de Valladolid)
}

Resumen. El presente artículo tiene una doble finalidad: (1) presentar la fundamentación teórica sobre la que se construye la transformación y posible mejora del Programa Integral de Deporte Escolar del Municipio de Segovia (PIDEMSG); y (2) describir pormenorizadamente los aspectos organizativos y la puesta en práctica del consejo asesor (CA). En esta investigación se ha utilizado la metodología comunicativa crítica, basada en los conceptos de acción dialógica y comunicativa, para la mejora del PIDEMSG a través del diálogo igualitario entre los diferentes agentes implicados. La aplicación de esta metodología se ha concretado en la creación y desarrollo de un CA, integrado por representantes de los diferentes colectivos que participan en el programa. A lo largo del curso 2013/14 se desarrollaron cuatro reuniones del CA en las que se aplicó la técnica del grupo de discusión comunicativo. El principal resultado obtenido ha sido la creación y desarrollo del CA. Por otro lado, las diferentes reuniones del CA permitieron identificar algunas propuestas de mejora que fueron implementadas de forma inmediata, durante ese mismo curso y los siguientes, como son: (1) la realización de un turno único de encuentros, (2) la creación de un blog del programa, (3) la inclusión del blog en la web del Instituto Municipal de Deportes de Segovia y (4) la elaboración de un libro de difusión. Se concluye que el CA se ha convertido en un órgano de referencia dentro del PIDEMSG que favorece la toma de decisiones futuras a partir del diálogo y el consenso entre los representantes de los diferentes colectivos.

Palabras clave. deporte escolar, programas de deporte escolar, evaluación de programas, metodología comunicativa crítica, consejo asesor, acción dialógica y comunicativa, diálogo igualitario.

\begin{abstract}
This paper has a dual purpose: (1) present the theoretical foundation which allows the transformation and possible improvement of the Segovia Local Authority Integrated Extracurricular Sport Programme (Programa Integral de Deporte Escolar del Municipio de Segovia (PIDEMSG)); and (2) describe in detail the organization and implementation of the advisory committee (AC). In this research we used the critical communicative methodology, based on the concepts of dialogic and communicative action for improving the PIDEMSG through egalitarian dialogue between several stakeholders. The application of this methodology has led to the creation and development of a AC, composed by several members who represent different groups involved in the programme. Throughout the year 2013/14 there have been four meetings of the AC where has been applied the technique of communicative focus group. The main result has been the creation and development of a AC. Thus, the different meetings have identified some improvement proposals which were implemented during the same year and the following years, such as: (1) the completion of a single round of Friday Games Nights, (2) the creation of a blog programme, (3) the inclusion of the blog on the website of Municipal Sports Institute and (4) the development of a book diffusion. We conclude that AC has become an organ of reference within PIDEMSG which helps making future decisions from the dialogue and consensus among different members.
\end{abstract}

Keywords. extracurricular sport, extracurricular sport programme, programme evaluation, critical communicative methodology, advisory committee, dialogic and communicative action, egalitarian dialogue.

\section{Introducción}

La sociedad actual se caracteriza por una necesidad de diálogo y participación ciudadana. Asimismo, en los últimos años se ha percibido un cambio en relación a cómo las personas nos relacionamos unas con otras (Aubert, García y Racionero, 2009). El éxito de las redes sociales como Twitter, Facebook, etc. o la irrupción de partidos políticos que promulgan los movimientos asamblearios y de participación ciudadana son algunas de las evidencias que muestran ese cambio y esa necesidad de diálogo y comunicación que demanda la sociedad.

Habermas (1987a:124) empleaba el término de acción comunicativa para referirse a «la interacción de al menos dos sujetos capaces de lenguaje y de acción que (ya sea con medios verbales o con medios extraverbales) entablan una relación interpersonal». Asimismo, los actores que formasen parte del acto comunicativo deberían buscar el entendimiento para coordinar de común acuerdo sus planes de acción, estableciéndose un diálogo entre iguales. Freire(1970) lo llamó acción dialógica, entendiendo el diálogo como una exigencia existencial y no como el mero acto de depositar ideas de un sujeto en otro o una discusión polémica entre dos sujetos que no aspiran a comprometerse con la búsqueda de la verdad. Por tanto, la acción dialógica requiere de amor, humildad, fe, esperanza y un pensar verdadero. «Si no amo el mundo, si no amo la vida, si no amo a los hombres, no me es posible el diálogo»; «Los hombres que carecen de humildad o aquellos que la pierden no pueden aproximarse al pueblo» (ib. 1970:101). «No hay diálogo, tampoco, si no existe una intensa fe en los hombres. Fe en su poder de hacer y rehacer. De crear y recrear. Fe en su vocación de ser más, que no es

Fecha recepción: 30-09-14- Fecha envío revisores: 30-09-14- Fecha de aceptación: 15-11-14 Borja Jiménez Herranz. borjajzhz@hotmail.com privilegio de algunos elegidos sino derecho de los hombres» (ib.1970:102). «Si los sujetos del diálogo nada esperan de su quehacer, ya no puede haber diálogo»; «Finalmente, no hay diálogo verdadero si no existe en sus sujetos un pensar verdadero» (ib.1970:103), un pensar crítico donde lo importante es «la permanente transformación de la realidad, con vistas a una permanente humanización de los hombres» (ib.1970:104).

Por consiguiente, una relación horizontal basada en la confianza entre los diferentes sujetos que intervienen en el acto comunicativo, será condición sine qua non para que se establezca un diálogo igualitario y productivo. «Las organizaciones humanas se rigen más que antes por razones, y menos por imposiciones; sin ir más lejos, obtienen mejores resultados las empresas que fomentan el diálogo horizontal favoreciendo los grupos cooperativos» (Gómez, Latorre, Sánchez y Flecha, 2006:39). En una sociedad cada vez más dialógica y participativa, resulta coherente que la investigación en Ciencias Sociales se adecúe a ella, favoreciendo las relaciones intersubjetivas orientadas al consenso y regidas por pretensiones de validez.

\section{Metodología Comunicativa Crítica}

Según Gómez et al (2006), frente a las perspectivas positivista, interpretativa y socio-crítica, surge una nueva formar de concebir y entender la realidad, la metodología comunicativa crítica. Estos autores consideran que la realidad social es de naturaleza comunicativa, dando importancia al diálogo y a la intersubjetividad, incluso para empezar a comunicarse los humanos: «Una mesa es una mesa porque es su día se llegó a ese acuerdo, porque es de manera consensuada como se le otorga significado intersubjetivamente» (Gómez et al. 2006:22). Asimismo, sostienen que esta metodología va más allá de las tres racionalidades descritas por Habermas (1984): Técnica (basada en el Interés Instrumental), Práctica (basada en el Interés Ético) y Crítica (basada en el Interés Emancipatorio), incorporando la racionalidad Comunicativa, en 
la que el objetivo no es alcanzar el éxito, sino el entendimiento. Se trata, por tanto, de alcanzar una acción comunicativa donde lo importante sean las argumentaciones basadas en pretensiones de validez y no de poder, donde la palabra del investigado tenga el mismo valor que la del investigador. Desde nuestro punto de vista, consideramos que esta metodología no constituye un nuevo paradigma, sino una evolución o nueva corriente del paradigma crítico, dado que se apoya claramente en el Interés Emancipatorio y busca una mayor justicia e igualdad social.

La metodología comunicativa crítica pretende describir la realidad, interpretarla y transformarla desde un Interés Emancipatorio, pero haciendo hincapié en cómo los significados se construyen comunicativamente a partir de interacciones entre las personas desde un plano horizontal. El objeto de estudio surge de las interpretaciones y reflexiones de las personas que participan en la investigación y que forman parte de la realidad social que se quiere transformar. De esta manera, el equipo investigador aporta la base teórica que es contrastada con el mundo de la vida (Habermas, 1987b), las vivencias y experiencias de las personas investigadas desde un diálogo igualitario (Flecha, 1997) y con las aportaciones de las diferentes personas que son consideradas en función de la validez de sus argumentos y no de la posición de poder de quienes las realizan.

Gómez et al. (2006) describen los siguientes postulados de la metodología comunicativa crítica: (a) Universalidad del lenguaje y de la acción. Todas las personas tienen la capacidad de comunicarse e interactuar con otras personas; (b) Las personas como agentes sociales transformadores. Las personas son capaces de interpretar la realidad, crear conocimiento y modificar sus estructuras; (c) Racionalidad comunicativa. Se utiliza el lenguaje como un medio de diálogo y de entendimiento; (d) Sentido común. Para conocer el porqué de las acciones hay que conocer el sentido común de las personas, el cual está determinado por la experiencia de la vida de cada persona y por el contexto socio-cultural; (e) Sinjerarquía interpretativa. Las interpretaciones de las personas investigadas tienen el mismo valor que las interpretaciones de los investigadores; (f) Igual nivel epistemológico. E investigador y las personas investigadas se sitúan en el mismo nivel. Solo desde la igualdad es posible comprender o explicar un fenómeno; (g) Conocimiento dialógico. Busca la transformación de la realidad social a través de la comunicación y el diálogo bajo pretensiones de validez y no de poder.

\section{El Programa Integral de Deporte Escolar del Municipio de Segovia y la necesidad de una transformación comunicativa y dialógica}

El PIDEMSG forma parte de un convenio de colaboración entre la Universidad de Valladolid (UVa) y el Instituto Municipal de Deportes de Segovia (IMDSG), que comenzó en el curso 2009-10, a partir del diagnóstico de la situación del deporte escolar en la ciudad (Gonzalo, 2012; Gonzalo, Manrique, Monjas y López, 2011; Pérez, 2011), y que posteriormente se amplió gracias al desarrollo del proyecto de $\mathrm{I}+\mathrm{D}+\mathrm{i}$ denominado: «Desarrollo de un Proyecto Integral del Deporte Escolar, para la franja de población 4-16 años en el municipio de Segovia durante el periodo 2011-2014». El PIDEMSG es un programa polideportivo, mixto y gratuito que es ofertado a todos los colegios del municipio (González, 2012). Anivel metodológico emplea un modelo comprensivo de iniciación deportiva(Thorpe, Bunker \& Almond, 1986), basado en los juegos modificados. En estos juegos se adaptan los diferentes elementos del juego deportivo estándar (materiales, espacios, reglas, etc.) a las características de los alumnos, con lo que se facilita así la comprensión de situaciones tácticas que constituirán una base sobre la que asentar posteriores aprendizajes técnicos. Cada centro cuenta con varios módulos polideportivos en función de la categoría: Actividad Física Jugada (AFJ) de 4 a 6 años, pre-benjamines de 6 a 8 años, benjamines de 8 a 10 años y alevines de 10 a 12 años. Todos los módulos cuentan con 2 horas de enseñanza durante la semana (2 días) y 1 encuentro los viernes por la tarde, con diferente periodicidad en función de la categoría. Los encuentros de los viernes tienen lugar en dos pabellones municipales donde se reúnen los alumnos de 6-8 colegios. El pabellón se divide en 2-3 espacios adaptados donde los diferentes grupos de alumnos realizan partidos cortos de unos 10 minutos. Transcurrido ese tiempo, se produce una rotación, de manera que al final del encuentro todos los grupos han conseguido jugar entre sí (Jiménez, López y Manrique, 2014).

El PIDEMSG favorece la formación de aquellos alumnos de la UVa que participan en el programa como monitores becados. Estos monitores suelen tener una escasa o nula experiencia en programas de deporte escolar (Manrique, Gea y Álvaro, 2013). Participan en el PIDEMSG gracias a una beca de colaboración y perciben por ello una retribución económica en función de las horas trabajadas. Además de los monitores becados, el programa también cuenta con la participación de 5 monitores contratados, graduados, diplomados o licenciados en educación, con amplia experiencia en programas de deporte escolar, con un contrato de 10 horas semanales y una duración de 8 meses. El trabajo de los monitores es regulado por dos coordinadores que cuentan con el apoyo y asesoramiento de cuatro profesores de la UVa (Martínez, Pérez y López, 2012).

Por otro lado, el programa trata de tener en cuenta otros aspectos relevantes para su buen funcionamiento y así atender todas las demandas educativas que se presentan, tales como la relación entre iniciación deportiva y valores (Ponce, 2012), la participación femenina (Escudero, 2012) o la integración de los alumnos inmigrantes que participan en el mismo (Carter, 2012).

Al final de cada curso se pasa un cuestionario muy estructurado entre los diferentes agentes implicados (alumnos, padres, monitores becados y profesores de educación física), para evaluar diferentes aspectos del programa (González, 2012; Hortal, 2012; Jiménez, 2013). Las conclusiones obtenidas a partir de todas estas investigaciones permiten la evaluación y mejora del programa de cara a futuras ediciones. Sin embargo, la toma de decisiones sobre qué aspectos deberían ser modificados ha recaído, desde el principio, en el equipo investigador, integrado por 4 profesores de la UVa, 2 coordinadores y 5 monitores contratados. Dado que se trata de un programa de deporte escolar municipal, parece necesario que la toma de decisiones sobre los aspectos del programa a modificar cuente con el consenso de todos los colectivos implicados y no sea una decisión exclusiva del equipo investigador. También es conveniente la participación del equipo administrativo de la UVa y del IMDSG, cuyas opiniones, experiencias y vivencias han de ser tenidas en cuenta de cara a la mejora del programa en futuras ediciones.

El convenio terminó en julio de 2014. De cara a la firma del nuevo convenio plurianual (2014-2018) era necesario contar con las opiniones, reflexiones y expectativas de los representantes de los diferentes colectivos implicados. Para agilizar y operativizar la toma de decisiones conjuntas, el equipo investigador de la UVa tomó la decisión de crear un consejo asesor (CA), un ente organizativo basado en la metodología comunicativa crítica (Gómez et al. 2006). El objetivo de este artículo es presentar las principales características del CAdel PIDEMSG así como la temporalización seguida, el tratamiento de los temas de debate que surgieron en las diferentes reuniones y las acciones puestas en marcha a lo largo del curso 2013-14.

\section{Metodología}

En la presente investigación se ha utilizado una metodología comunicativa crítica, buscando el diálogo igualitario y la intersubjetividad entre los diferentes colectivos implicados, reconociendo la diversidad de perspectivas y vivencias y favoreciendo las pretensiones de valor sobre las de poder. La organización comunicativa crítica se ha conseguido gracias a la creación del CA (Martín y Ríos, 2014).

El consejo asesor estaría formado por personas representativas de colectivos, grupos y/o comunidades de la población participante en la investigación, reconociendo la riqueza de sus diversas aportaciones y maneras de entender y transformar la realidad. Entre las funciones de este órgano asesor se pueden destacar: su aporte de conocimientos, la revisión crítica de los documentos, la 
orientación sobre el desarrollo y proceso del proyecto, el control de que éste sea comunicativo, la valoración de toda la investigación incluyendo sus conclusiones y, especialmente, que los resultados obtenidos contribuyan a mejorar la realidad de los colectivos a los que va dirigido el estudio. (Gómez et al. 2006:48).

La creación de este CA tenía una doble finalidad: (a) hacer avanzar el proyecto de investigación a través del uso de metodologías participativas; y (b) asesorar al equipo investigador mediante la toma de decisiones conjuntas, para mejorar el programa, de cara a la firma del nuevo convenio de colaboración entre el IMDSG y la UVa. Además de orientar sobre el desarrollo del proyecto, el CA también valoró las conclusiones finales que se obtuvieron y procuró que éstas contribuyesen a mejorar la realidad social del PIDEMSG Las características del CA se explican en los siguientes apartados.

\section{Participantes}

A la hora de configurar el CA, se pretendió que en él estuvieran representados todos los colectivos que, de forma directa o indirecta, están implicados en el programa. Por ello, en primer lugar se definieron los diferentes roles que integrarían el CA y se elaboró una lista de posibles candidatos en función del rol que desempeñan. Seguidamente se contactó con las diferentes personas para conocer su predisposición o no a participar en la investigación. Para ello se utilizaron diferentes canales como la llamada telefónica, el correo electrónico y la comunicación cara a cara. Las personas que finalmente aceptaron participar en la investigación firmaron un contrato de colaboración en la primera reunión, asumiendo el papel de representantes de su colectivo y comprometiéndose a asistir y participar activamente en las diferentes reuniones. Asimismo, el contrato también garantizaba el anonimato y la confidencialidad de los datos (ver figura 1 ).

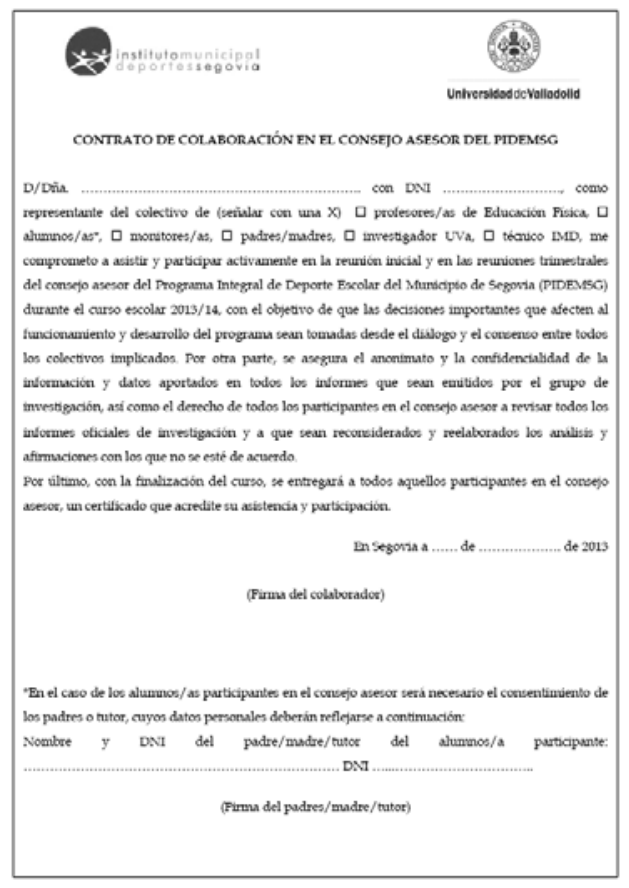

Fi gura 1. Contrato de colaboración

Los integrantes del CA a lo largo del curso 2013-14 fueron: 2 alumnos y 1 alumna, 2 maestros de educación física, 2 madres y 1 padre, 1 monitor y 1 monitora becados, 1 monitor (hacía a la vez de secretario) y 1 monitora contratados, 1 coordinador del programa, 2 profesores de la UVa que forman parte del equipo investigador, 1 técnico del IMDSG 1 miembro de la administración de la UVa y 1 coordinador o moderador del CA (ver tabla 1).

Como se puede observar en la tabla 1 , no siempre todos los participantes asistieron a todas las reuniones, en unas ocasiones por incompatibilidad de horarios y en otras porque sólo se requería la presencia
Tabla 1.

Participantes del consejo asesor y asistencia a las reuniones

\begin{tabular}{|c|c|c|c|c|}
\hline Miembros & $1^{1^{a} \text { reunión }}$ & $2^{2^{a} \text { reunión }}$ & $3^{a}$ reunión & $4^{a}$ reuniór \\
\hline Al umno 1 & $\mathrm{x}$ & A & A & A \\
\hline Al umno 2 & A & A & A & $\mathrm{x}$ \\
\hline Al umna & A & A & A & A \\
\hline Maestro EF 1 & A & A & A & A \\
\hline Maestro EF 2 & A & A & A & $\mathrm{x}$ \\
\hline Madre 1 & A & A & A & A \\
\hline Madre 2 & A & A & A & $\mathrm{x}$ \\
\hline Padre & $\mathrm{x}$ & $\mathrm{X}$ & A & $\mathrm{x}$ \\
\hline Monitor becado & A & A & A & A \\
\hline Monitora becada & A & $\mathrm{x}$ & A & $\mathrm{x}$ \\
\hline Monitora contrat ada & A & A & A & A \\
\hline Monitor contratado (secretario) & A & A & A & A \\
\hline Coordinador PIDEMSG & A & A & A & A \\
\hline Profesor UVa 1 & A & A & $\mathrm{x}$ & A \\
\hline Profesor UVa 2 & $\mathrm{x}$ & $\mathrm{x}$ & A & A \\
\hline Técn ico IMDSG & A & $\mathrm{x}$ & A & A \\
\hline Miembro Admi nistrativo UVa & $\mathrm{x}$ & A & $\mathrm{x}$ & $\mathrm{x}$ \\
\hline Coordinador consejo asesor & A & A & A & A \\
\hline TOTAL ASISTENTES & 13 & 14 & 16 & 12 \\
\hline
\end{tabular}

A = siste a la reunión. $X=$ No asistea la ieunín

puntual de un representante. En cualquier caso, siempre se procuró que hubiese, al menos, un miembro representando a cada colectivo. Asimismo, para garantizar el anonimato y la confidencialidad de los datos al que hacía referencia el contrato de colaboración, se decidió identificar a cada miembro con un código en cada una de las reuniones. Cada código se estructura en dos partes: la primera hace referencia al número de la reunión y la segunda al miembro que forma parte del CA. Por ejemplo, el código CA3-MEF1 hace referencia al maestro de educación física número 1(MEF1) que intervino en la tercera reunión del CA(CA3). De igual manera, para identificar a los alumnos se utilizan las siglas «Al», para las madres y padres «M/Pad», los monitores becados «MonA», los monitores contratados «MonC», el coordinador «Coor», los profesores de la universidad «Inv», el técnico del Ayuntamiento «IMD», el miembro administrativo UVa «Adm» y el coordinador del consejo asesor «CAA».

\section{Técnicas de recolección de datos}

La técnica empleada para la recogida de los datos en las cuatro reuniones del CA fue el «Grupo de Discusión Comunicativo». Ésta es unas de las técnicas cualitativas de orientación comunicativa descritas por Gómez et al. (2006).

Según estos autores, el grupo de discusión comunicativo supone un diálogo igualitario entre varias personas pertenecientes al grupo o comunidad objeto de investigación. El grupo elegido es natural, es decir, está compuesto por personas que pueden haberse conocido con anterioridad y desarrollar diferentes actividades. Las reuniones se realizan en un contexto conocido por los participantes. La persona investigadora que coordina o modera el grupo de discusión comunicativo es un participante más en el grupo, aportando sus conocimientos y compartiéndolos en plano de igualdad. Se elabora un guion o un orden del día, a partir del marco teórico y de los objetivos del estudio, para marcar los temas a tratar, los cuales son conocidos por el grupo de antemano y no son estancos, sino que pueden ampliarse y abarcar otras esferas que en un principio no se habían contemplado. Se trata pues de una técnica abierta y flexible, que busca el diálogo igualitario y la intersubjetividad entre los miembros de los diferentes colectivos con la intención de mejorar su realidad social.

Durante el curso 2013-14 se desarrollaron 4 reuniones del CA (ver tabla 2) donde se aplicó el grupo de discusión comunicativo. Todas esas reuniones tuvieron lugar en una de las aulas de la universidad. La información sobre la fecha, hora, lugar de celebración y orden del día de cada una de las reuniones era facilitada a los miembros del CA por diferentes vías (correo electrónico, SMS y WhatsApp). Los mismos canales eran utilizadas por los miembros para confirmar su asistencia o no a la reunión.

La primera reunión, realizada el 5 de noviembre de 2013, sirvió para explicar a los miembros qué es un CA, cómo funciona el grupo de discusión comunicativo y qué se pretendía alcanzar con las diferentes reuniones. Además de esta explicación inicial sobre las razones y el porqué de crear un CA, también se abordaron cuáles eran los puntos 
fuertes del programa, los puntos débiles y las posibles soluciones a esos puntos débiles identificados. De esta manera se pretendía tener una visión global de cómo los diferentes colectivos percibían el programa.

Un mes más tarde, el 10 de diciembre, se celebróla segunda reunión. Se dedicó a la búsqueda de acciones concretas y a las posibles alternativas que contribuyesen a solucionar los problemas detectados en la primera reunión.

La tercera de las reuniones tuvo lugar el 25 de marzo de 2014. En ella se analizaron las diferentes medidas a tomar para dar solución a otro de los problemas detectados por el CA, la escasa difusión del programa y, por tanto, el poco conocimiento del mismo por una parte importante de la población del municipio.

La cuarta y última reunión fue el 3 de junio. Allí se presentaron las principales conclusiones a las que se había llegado tras las tres reuniones anteriores, dentro de las cuales se incluían las acciones que ya se habían puesto en funcionamiento y las futuras acciones que se llevaron a la firma del siguiente convenio para que fueran tenidas en consideración.

Tabla 2

\begin{tabular}{|c|c|c|}
\hline Reunión & Fecha & Objetivo \\
\hline $1^{\text {a }}$ reunión & $5 / X \mathrm{X} / 2013$ & $\begin{array}{l}\text { Conocer los objetivos, funciones y organización del cons ejo asesor. } \\
\text { Identificar las fortalezas del programa sus debilidades y las } \\
\text { posibilidades de mejora. }\end{array}$ \\
\hline $2^{\mathrm{a}}$ reunión & 10/XII/2013 & $\begin{array}{l}\text { Buscar acciones concretas y posibles que den respuesta a las } \\
\text { debilidades detectadas en la primera reunión. }\end{array}$ \\
\hline $3^{\text {a }}$ reunión & 25/III/2014 & $\begin{array}{l}\text { Analizar las diferentes propuestas para la mejora de la difusión del } \\
\text { programa. }\end{array}$ \\
\hline $4^{a}$ reunión & $3 / \mathrm{VI} / 2014$ & $\begin{array}{l}\text { Presentar las concl usiones obtenidas en las reuniones anterio ies. } \\
\text { Conocer las acciones que ya se han llevado a cabo y las que se } \\
\text { trasladarán a la firma del próximo convenio. }\end{array}$ \\
\hline
\end{tabular}

\section{Procedimientos y análisis de los datos}

Todas las reuniones fueron grabadas en audio bajo el consentimiento de los miembros del CA. Después de cada reunión, la grabación era transcrita en una hoja de texto y ésta analizada posteriormente con el programa Atlas.ti, versión 7.0.83. Para cada una de las reuniones se elaboró una unidad hermenéutica donde se incluían las redes de comentarios o «networks» que nos permitían representar gráficamente las relaciones entre categorías y subcategorías (ver figura 2).

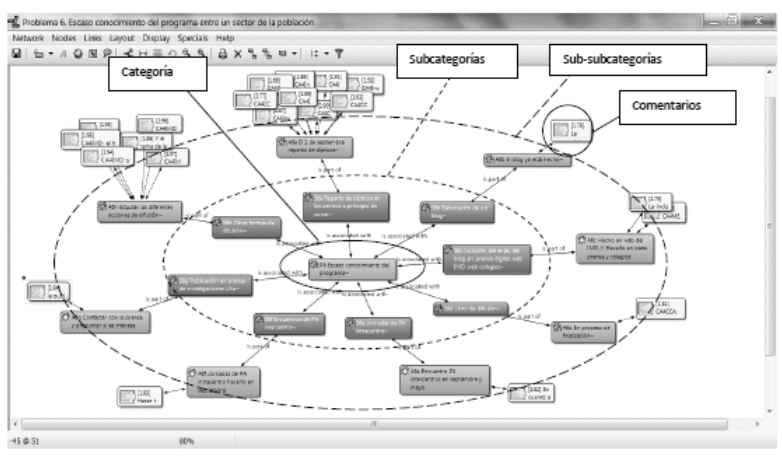

Figura 2. Red de comentarios sobre una de las categorías abordada en la cuarta reunión

Como se puede observar en el ejemplo de la Figura 2, una categoría (una de las problemáticas detectadas) se relaciona con una serie de subcategorías (las posibles alternativas que se trataron para dar solución al problema) y éstas a su vez con otras sub-subcategorías (las acciones concretas que ya se han puesto en marcha o se llevarán a la firma del próximo convenio), las cuales están vinculadas con comentarios literales realizados en la reunión.

\section{Resultados}

El principal resultado obtenido en este proyecto ha sido la creación y el desarrollo exitoso de un CA, en el cual están representados los intereses y vivencias de los diferentes colectivos implicados en el PIDEMSG. A lo largo de este primer año de funcionamiento, todos los miembros han podido expresarse con total libertad, debido al carácter horizontal de las interacciones que se dan dentro del grupo de discusión comunicativo y han adquirido un conocimiento más profundo y real de lo que, en realidad, es el programa. Además, todas las intervenciones surgidas en las diferentes reuniones han tenido un objetivo común: la mejora y progreso del programa. Asimismo, si el coordinador del CA detectaba que algún grupo o representante de grupo no había intervenido, les preguntaba directamente para conocer su opinión sobre el tema. Esto ocurrió algunas veces con los representantes de los alumnos de Educación Primaria.

Las cuatro reuniones realizadas a lo largo del curso 2013-14 nos han permitido detectar importantes acciones de mejora que han sido asumidas por el equipo investigador y por los diferentes colectivos implicados en el programa. Posteriormente, se introdujeron en el nuevo convenio entre la UVa y el IMDSG (2014-2017). A continuación vamos a describir aquellas medidas que fueron implementadas a lo largo del curso 2013-14.

Una de las medidas sugeridas por el CA y aplicada a lo largo del curso fue la realización de un único turno de encuentros por categoría. Anteriormente se realizaban dos turnos de encuentros por categoría. El primer turno, al que acudían los alumnos de 6-8 colegios, se realizaba de 16:00 a 18:00 y el segundo turno, con los alumnos de los otros 6-8 colegios restantes, de 18:00 a 20:00. Esta medida surgió ante el escaso número de alumnos que acudían al segundo de los turnos, resultando complicado hacer rotaciones en los diferentes partidos y, por tanto, perdiendo parte del sentido de los encuentros. A partir del curso 201314, todos los alumnos de todos los colegios de la misma categoría asistieron a un único turno de 17:00 a 19:00, favoreciendo así la asistencia de un mayor número de participantes en el encuentro y con la posibilidad de enriquecer las rotaciones de los diferentes equipos.

Yo creo que tenemos que avanzar hacia un encuentro único por categoría. Lo de hacer dos encuentros está visto que no funciona. (CA2Inv1).

Nosotros sabemos más o menos que tal persona va a ir a esta hora porque le conozco de que va a esta hora. Pero no sabemos los de la otra hora (...). Porque lo de los dos horarios surge porque todos juntos no cabemos. Pero por probar un día todos los centros y... «pues sí es verdad que funciona», entonces pues sí que se podría hacer. (CA2Al3).

Yo creo que es mejor que hagamos unúnico turno, aunque haya más tiempo de espera o aunque los niños participen menos. (CA2MonC2).

Otra de las medidas que contó con la aprobación del CA fue la creación de un blog con la intención de mejorar la difusión del programa y que éste llegase al mayor número de personas posible. A lo largo del curso, dos miembros del equipo investigador se encargaron del diseño y elaboración del blog. En él se puede consultar una gran cantidad de información: miembros que lo integran, finalidad, historia del programa, publicaciones, investigaciones, calendarios de encuentros, etc. Por lo tanto, se puede consultar, de forma rápida y sencilla, contenidos muy útiles para usuarios e investigadores del Programa. Para más información, acerca de sus contenidos, puede consultarse el blog a través del siguiente enlace: http://grupoinvesdeporteescolarsg.wordpress.com/

Hemos creado un blog en el que viene recogido todo lo que hay publicado sobre el programa. (CA3MonC1).

En el blog hay varias pestañas: una de «inicio» donde viene una presentación de lo que es el programa, otra de «quienes somos» donde aparece el equipo que está trabajando (...) otra pestaña de «consejo asesor» en el que aparece lo que estamos haciendo (...). Es una manera sencilla de informar a la gente que aún no conoce el programa (...). También encontramos algo interesante para los padres y para los alumnos, como son los calendarios de los encuentros, ya que muchas veces les das los folios de los calendarios y o se pierden o se quedan en las mochilas (CA3CCA).

La tercera medida puesta en funcionamiento fue la inclusión de la dirección del blog en la página web del IMDSG. Al incluir el blog en una web frecuentemente consultada por gran parte de la población del municipio, se favorece que un mayor número de usuarios accedan al blog del programa y, por lo tanto, se contribuya a la difusión del mismo. 
Yo creo que todo lo que sea información (...) todo lo que sea difundir información, cualquiera de los medios, yo creo que habría que hacerlo. Todo suma. (CA3IMD).

En la web del IMDSG ya hay un enlace del blog del programa. (CA3Inv2).

De cara al futuro inmediato, y a propuesta del CA, se pretende la inclusión del blog en las web de los centros escolares y de la prensa digital.

Yo creo que el enlace del blog ya podría estar en la web de todos los centros (CA3MonC1).

No hay ningún problema para incluir el enlace del blog en la web del centro. (CA4MEF1).

Sí que se puede incluir el enlace en la prensa digital. Pero, ¿̇a qué paginas vas? Yo te diría otra vez, a las páginas de los periódicos. No páginas específicas de deporte, científicas, de grupos de colectivos, sino que sean las que más difusión tienen que son los periódicos digitales. Ahora mismo hay que meterse. (CA3Inv2).

Por último, la cuarta propuesta aprobada por el CA fue la realización de un libro de difusión. Este libro está coordinado por tres miembros del grupo investigador y en él participan todas aquellas personas que han trabajado o investigado sobre el PIDEMSG. Con la edición de este libro se pretende explicar las principales características y señas de identidad del PIDEMSG a través de un lenguaje sencillo y accesible a toda la población. Mediante esta propuesta se favorece nuevamente la mejora de la difusión del programa, uno de los principales problemas detectados en la primera reunión del CA.

Es un libro que partió de una idea de otro componente que también es coordinador del libro. Redactó un índice y se quedó ahí. Y ahora lo estamos llevando a cabo. Explica un poco los principios del programa, qué métodos utiliza en la enseñanza, los agentes implicados en el programa, etc. (CA3MonC1).

El libro tiene un objetivo muy claro: que sirva de difusión del programa. El lenguaje es sencillo y cercano a cualquier persona que no sea una entendida en la materia de la EF (...). Los autores que están escribiendo el libro son todos aquellos compañeros que están hoy en el programa de deporte escolar y todos aquellos que han estado en años anteriores. (...) Y bueno, pues va a ser una muestra, yo creo que más que significativa, de lo que se ha hecho durante todo este tiempo. (CA3Coor).

Lo que se ha querido con el presupuesto que teníamos era, aumentar el número de copias para llegar al máximo número de personas posible. Y luego pues concretar mucho para intentar plasmar todo el programa y la evolución que ha habido en 80 páginas. (CA3MonC1).

\section{Discusión}

Tal y como hemos podido comprobar a lo largo del artículo, los conceptos de acción comunicativa (Habermas, 1987) y de acción dialógica (Freire, 1970) tienen cabida y lugar de aplicación dentro de la metodología comunicativa crítica, donde el diálogo igualitario y las relaciones intersubjetivas basadas en pretensiones de valor constituyen la base fundamental sobre la que crear conocimiento, transformando la sociedad actual en busca de colectividades más justas y democráticas (Flecha, 1997; Gómez et al. 2006).

El principal resultado obtenido en esta investigación ha sido la creación y el desarrollo exitoso del CAdel PIDEMSG. En las diferentes reuniones del CA se aplicó la técnica del grupo de discusión comunicativo donde, a través del diálogo, se llegaba a interpretaciones y conclusiones que en reuniones posteriores eran nuevamente debatidas y consensuadas definitivamente (Gómez et al. 2006). Por tanto, era fundamental que hubiese una coherencia interna entre unas reuniones y otras. Para la puesta en práctica de esta metodología comunicativa crítica es necesario tener en cuenta las acciones y requisitos propuestos por Flecha, Vargas y Dávila (2004): (1) el respeto de todas las opiniones y puntos de vista, (2) la importancia de los argumentos como medios para llegar a acuerdos, (3) la necesidad de que las personas investigadas participen en un plano de igualdad con respecto a las personas investigadoras y (4) la búsqueda de la transformación social a través de la acción comunicativa y mediante el diálogo. Todos y cada uno de estos requisitos fueron respetados a lo largo de las cuatro reuniones.

Otro de los resultados de esta investigación ha sido la decisión de realizar un único turno de encuentro por categoría. Con esta decisión se pretende que haya una mayor concentración de niños en un espacio de tiempo de dos horas, posibilitando la realización de un mayor número de rotaciones entre los diferentes equipos y, por tanto, aumentando el número de interacciones sociales y los procesos de socialización (Gutiérrez, 1995)

Por otro lado, los resultados relativos a la creación de un blog del programa y su inclusión en diferentes páginas web(IMDSG, colegios y prensa digital), así como la elaboración de un libro de difusión, son diferentes medidas que fueron aprobadas y consensuadas por los miembros del CA, con la intención de aumentar el conocimiento del programa entre la población del municipio. En este sentido, sería interesante establecer un programa de difusión (Mestre y García, 1997) en el que además de estas dos medidas, se incluyeran otras nuevas que permitieran impulsar el programa y darle a conocer a un mayor número de personas, como por ejemplo, la presencia del programa en redes sociales como Facebook o Twitter.

El hecho de trabajar desde la horizontalidad y la igualdad en el diálogo ha chocado frontalmente con las ideas preconcebidas, prejuicios y creencias de muchos miembros del CA, que tradicionalmente han supuesto que la palabra del «experto» valía más que la del «no experto». Esto se ha podido observar en las reuniones iniciales del CA, donde algunos miembros no entraban a cuestionar las ideas u opiniones de los investigadores u otros miembros «cualificados» y, en cambio, se mostraban inseguros al exponer sus contadas ideas o sugerencias, aunque éstas estuviesen correctamente argumentadas. Tal y como concluyen Arandia, Alonso-Olea y Martínez-Domínguez (2010), esto es algo que el alumnado ha aprendido en su vida académica, donde la palabra del profesor no era cuestionada ni puesta en entredicho y que ha seguido reproduciendo en la vida adulta. Parece necesario romper con estas creencias y favorecer, desde edades tempranas, el diálogo igualitario y el pensamiento crítico. Castro, Gómez y Macazaga (2014) afirman que la interacción será imprescindible para que se produzcan procesos de transformación individual y social a través del diálogo. Mediante la puesta en práctica y desarrollo del CA, los diferentes miembros han ido comprendiendo que sus intervenciones y aportaciones son igual de válidas que las del resto de miembros, siempre y cuando estén debidamente argumentadas. Para ello ha sido necesario un proceso de transformación interior, donde la persona se ha desprendido de aquellas creencias no igualitarias que formaron parte de su currículum oculto. Así, en las últimas reuniones, quizás como resultado de la confianza surgida entre los diferentes miembros, se observó una mayor frecuencia de intervenciones y con mayor seguridad por parte de los representantes de monitores becados, de madres y padres y de alumnos, aunque éstos últimos, en ocasiones, tuviesen que ser preguntados directamente por el coordinador del CA u otros miembros. Como sostiene Martins (2006), el cambio social sólo es posible con el cambio interior personal, rompiendo con los valores de poder todavía arraigados en nosotros a través de la práctica del diálogo igualitario. Por último, debemos destacar las interacciones y la participación de la comunidad como dos factores clave para favorecer el aprendizaje en la actual sociedad (Martín y Ríos, 2014). Estos factores han sido ampliamente desarrollados en las sucesivas reuniones del CA y nos han permitido conocer las futuras líneas de actuación sobre las que se apoyará el programa.

\section{Conclusiones}

A lo largo del artículo hemos podido conocer las principales características del CA del PIDEMSG: cuáles son los postulados metodológicos sobre los que se asienta, quiénes son los miembros que lo integran, cómo se crea y organiza y cuál es la finalidad de su creación. También se ha incidido en la temporalización de las reuniones y en el tratamiento de los 
temas de debate, resultando fundamental la existencia de una coherencia interna que permita interrelacionar las diferentes reuniones. Por último, se han mostrado, a modo de resultados, las acciones que ya fueron puestas en marcha a lo largo del curso 2013-14 con la intención de mejorar el programa.

Esperamos que este artículo pueda ser de utilidad a las instituciones y personas implicadas en programas de deporte escolar municipal con un claro enfoque participativo, polideportivo y educativo. Asimismo, este artículo también puede ser de interés para todas aquellas personas que estén interesadas en la puesta en práctica de la metodología comunicativa crítica en diferentes programas y actividades educativas. Como prospectiva de futuro, se pretende dar a conocer las principales conclusiones obtenidas en las diferentes reuniones del CA que fueron llevadas a la firma del nuevo convenio entre la Fundación Parque Científico, la UVa y el IMDSG.

\section{Agradecimientos}

Este estudio es parte del proyecto I+D+i denominado: «Desarrolloy evaluación del Programa Integral del Deporte en Edad Escolar en la ciudad de Segovia para la franja de edad de 4-16 años en la ciudad de Segovia (2011-2014)», desarrollado a través de un convenio de I+D entre el Instituto Municipal de Deportes del Excmo. Ayuntamiento de Segovia y la Universidad Valladolid, con una duración de 4 años (20112014).

Agradecemos su colaboración a todas las personas que han participado en el Consejo Asesor durante el curso 2013-2014.

\section{Bibliografía}

Arandia, M., Alonso-Olea, M. J., y Martínez-Dominguez, I. (2010). La metodología dialógica en las aulas universitarias. Revista de educación, 352, 309-329.

Aubert,A., García, C., y Racionero, S. (2009). El aprendizaje dialógico. Cultura y Educación, 21 (2), 129-139.

Carter, B. I. (2012). La integración del alumnado inmigrante en el programa de deporte escolar de Segovia. Trabajo fin de máster. Valladolid: Universidad de Valladolid. http://uvadoc.uva.es/ bitstream/10324/1602/1/TFM-B.14.pdf

Castro, M., Gómez,A., y Macazaga,A. M.(2014).Aprendizaje dialógico y grupos interactivos en educación física. Retos, Nuevas tendencias en Educación Física, Deportey Recreación, 25, 174-179.

Escudero, J. A. (2012). La participación femenina en el Programa Integral de Deporte Escolar del Municipio de Segovia. Trabajo fin demáster. Valladolid: Universidad de Valladolid. http://uvadoc.uva.es/ bitstream/10324/1886/1/TFM-B.29.pdf

Flecha, R. (1997). Compartiendo palabras. El aprendizaje de las personas adultas a través del diálogo. Barcelona: Paidós.

Flechas, R., Vargas, J., y Dávila, A. (2004). Metodología comunicativa crítica en la investigación de las ciencias sociales: La investigación Warkaló. Lan Harremanak, 11, 21-33.

Freire, P. (1970). Pedagogía del oprimido. Madrid: Siglo XXI.

Gutiérrez, S. (1995). Valores sociales y deporte. Madrid: Editorial Gymnos.

Gómez, J., Latorre, A., Sánchez, M., y Flecha, R. (2006). Metodología comunicativa crítica. Barcelona: El Roure.

González, M. (2012). Evaluación del funcionamiento del primer curso de implantación de un programa integral de deporte escolar en educación primaria en el municipio de Segovia. Tesis doctoral. Valladolid: Universidad de Valladolid. http://uvadoc.uva.es/ bitstream/10324/1025/1/TESIS186-120625.pdf

González, M., Manrique, J.C., y López, V. M. (2012). Valoración del primer curso de implantación de un programa municipal integral de deporte escolar. Retos. Nuevas tendencias en Educación Física, Deportey Recreación, 21,14-18. http://www.retos.org/numero_21/ Retos\%2021\%2014-18.pdf
Gonzalo, A., Manrique, J.C., Monjas, R. y López, V.M. (2011). Diagnóstico de la situación del deporte escolar en la ciudad de Segovia. Análisis de la información obtenida a través de los cuestionarios realizados a los profesores de educación física. Segovia: E.U. Magisterio de Segovia. Universidad de Valladolid. (e-book).

Gonzalo, L.A. (2012). Diagnóstico de la situación del deporte en edad escolar en la ciudad de Segovia. Tesis doctoral. Valladolid: Universidad de Valladolid.

Habermas, J.(1984). Teoría de la acción comunicativa: Complementos y estudios previos. Madrid: Cátedra.

Habermas, J. (1987a). Teoría de la acción comunicativa. I: Racionalidad de la acción y racionalización social. Madrid: Taurus.

Habermas, J. (1987b). Teoría de la acción comunicativa. II: Crítica de la razón funcionalista. Madrid: Taurus.

Hortal, R. M. (2012). Evaluación del funcionamiento del Programa Integral de Deporte Escolar durante el curso 2010-2011. Trabajo fin de máster. Valladolid: Universidad de Valladolid. http:// uvadoc.uva.es/bitstream/10324/1613/1/TFM-B.25.pdf

Jiménez, B. (2013). Evaluación del tercer curso de funcionamiento del Programa Integral en el Municipio de Segovia (2011/12). Trabajo fin de máster. Valladolid: Universidad de Valladolid. http:// uvadoc.uva.es/bitstream/10324/3208/1/TFM-B.38.pdf

Jiménez, B., López, V.M., y Manrique, J.C. (2014). Evaluación comparativa de resultados de un programa municipal de deporte escolar. Retos. Nuevas tendencias en Educación Física, Deporte y Recreación, 26, 15-20.

Manrique, J.C., Gea, J.M., y Álvaro, M. (2013). Perfil y expectativas del técnico de deporte escolar en el municipio de Segovia (España). Revista Internacional de Medicina y Ciencias de la Actividad Física y el Deporte, 13 (50), 367-387.

Martín, M., y Ríos, O. (2014). Prevención y resolución de conflicto en educación física desde la perspectiva del alumnado. Retos, Nuevas tendencias en Educación Física, Deporte y Recreación, 25, 162167.

Martínez, S., Pérez, D., y López, V.M. (2012). Diseño y desarrollo de un programa integral de deporte escolar municipal. Revista Pedagógica $A D A L, 15$ (24), 7-13.

Martins, D. (2006). Comprensión crítica y aprendizaje dialógico: lectura dialógica. Lectura y vida, 27 (1), 18-28.

Mestre, J. A., y García, E. (1997). La gestión del deporte municipal. Barcelona: INDE.

Pérez, D. (2011). Buscando un modelo de deporte escolar para el municipio de Segovia. Un estudio a partir de las valoraciones, intereses y actitudes de sus agentes implicados. Tesis doctoral. Valladolid: Universidad de Valladolid.

Ponce, A. (2012). La transferencia de valores a través de la práctica deportiva. Un estudio de caso: la transferencia entre el programa de deporte escolar de la ciudad de Segovia y el deporte federado. Trabajo fin de máster. Valladolid: Universidad de Valladolid. http:/ /uvadoc.uva.es/bitstream/10324/1596/1/TFM-B..10.pdf

Thorpe, R., Bunker, D., \& Almond, L. (1986). Rethinking games teaching. Loughborough: University of Technology, Loughborough

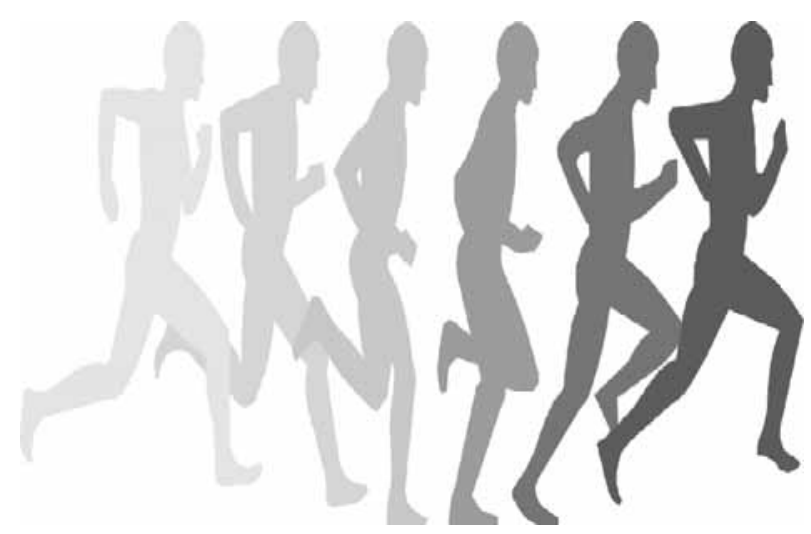

